\title{
An insight on the (Hindu's) unified quantum cosmology
}

\author{
U. V. S. Seshavatharam ${ }^{1 *}$, S. Lakshminarayana ${ }^{2}$ \\ ${ }^{1}$ Honorary faculty, I-SERVE, Alakapuri, Hyderabad-35,Telangana, India \\ ${ }^{2}$ Dept. of Nuclear Physics, Andhra University, Visakhapatnam-03,AP, India \\ *Corresponding author E-mail:seshavatharam.uvs@gmail.com
}

\begin{abstract}
In the heuristic approach, with reference to 'conservation of energy', 'initial light speed expansion', 'continuous light speed rotation', 'Kerr-Schwarzschild radius', 'conservation of centripetal force', 'Planck scale', 'quantum gravity' and 'Hindu model of cosmic age', we introduce a heuristic 'model of cosmology'. The authors would like to stress the fact that, 'with light speed rotation' qualitatively 'Hubble parameter' and 'angular velocity' both can be shown to be secondary physical constants and their individual roles can be shown to be similar. With five unified, simplified and workable assumptions, a number of useful cosmological formulae can be generated. With reference to current microwave back ground temperature obtained magnitude of current Hubble parameter is accurately fitting with the recommended value. With the proposed assumptions:1) the intended purpose of 'lambda' term can be understood and in future it can be relinquished. 2) Cosmic acceleration and dark energy concepts can be relinquished at fundamental level. 3) Cosmic flatness can be well understood.4) Comic 'horizon problem' can be eliminated at fundamental level. In future, either from 'academic interest' point of view or from 'serious research' point of view, this model can be recommended for in depth analysis at fundamental level.
\end{abstract}

Keywords: Planck Scale; Modern Cosmology; Hindu Cosmology; Initial Light Speed Expansion; Very Slow Deceleration; Continuous Light Speed Rotation; Cosmic Temperature; Quantum Gravity.

\section{Introduction}

With 'very large cosmic size' and 'very large cosmic time' it is certainly possible to understand the current universe. In this review paper, by modifying the early proposed assumption- 1 and by introducing assumption-5, the authors revised their recent paper (U. V. S. Seshavatharam, Lakshminarayana. S 2015). With reference to Hindu model of cosmology (Ebenezer Burgess 1860, KedarNathShukla 2014), age of the current universe is around 158.7 trillion years and total cosmic age is 311.4 trillion years. By considering the new assumption 5 , in a most scientific way, the authors tried to fit the current age of the universe with a value of 310 trillion years. This is for further research and analysis. Considering the idea of 'initial light speed expansion with very slow reduction in cosmic expansion speed', by this time it is possible to show that, current universe is expanding with a speed of c/146 with very minute deceleration. It may be noted that, size being very large (i.e.146 times of Hubble radius) if current universe is very slowly decelerating, then it apparently resembles 'uniform rate' of expansion and this proposal can be compared with the recent type- Ia super novae observational conclusions (Nielsen. J.T et al 2015).

Very recently, by vigorously analyzing the super novae type Ia data, Nielsen. J.T et al,in a paper posted in arXivon 3rdJune 2015 suggest that, at present universe seems to be expanding at constant rate (Jun-Jibe Wei et al 2015, F. Melina and R. S. Maier 2013) and evidence for cosmic acceleration is only marginal. In 2013, Abhas Mithra suggested that, the currently believed "Cosmic acceleration" could be an artifact of in homogeneity (A. Mitra,2013, A. MITRE et al 2013). In 2011, Paul J. Steinhardt, one of the crea- tors of the inflation theory, suggested against to "Inflation" (Steinhardt, P.J 2011). These published papers seriously cast doubt on the basics and advanced concepts of modern cosmology. From unification point of view S.W. Hawking expected quantum cosmology (S.W. Hawking 1987). By following the Schwarzschild formula (W.M. Stuckey 1994) and other basic and reasonable assumptions, our recently published paper (Tatum, E. T et al 2015) titled with "The basics of flat space cosmology" discounts the need for dark energy (Peebles, P. J et al 2003), the theory of cosmic inflation (Goth, A.H 1997, 1981) and Horizon problem entirely.

It is not a surprise to say that, 'nature loves symmetry'. All the celestial objects are found to be rotating. If universe is 'really an expanding sphere', then it is very natural to have some angular momentum (Siva ram, C and Arum 2012). In that case, it is absolutely wrong to say that, "subject of cosmology can be developed and understood without cosmic rotation". If universe is "really not rotating', it is also absolutely wrong to say that, "Subject of cosmology can be developed and understood with cosmic rotation". Since 1920 cosmologists are trying to understand the observable universe, in all the possible versions. The very important point to be noted is that, subject of cosmology is mostly subjected to very long range cosmological observations and are beyond the scope of confirmation. As universe is vast, time to time observations are indicating different set ofresults and are again subjected to future observations. By going through the history of observational cosmology one can understand this. It's very surprising to say that, recent observations indicate that our galaxy size is $50 \%$ larger than we believe (Yan CSU et al 2015). It is well believed that our universe constitutes so many large galaxies. If so, one cannot make a clear cut comment on the current size of the universe. In this paper, 
the authors would like to stress the fact that, 'with light speed rotation' qualitatively 'Hubble parameter' and 'angular velocity' both can be shown to be secondary physical constants and their individual roles can be shown to be similar.

In a heuristic approach, with reference to 'conservation of energy', 'initial light speed expansion', 'continuous light speed rotation', 'Kerr-Schwarzschild radius', 'constancy of centripetal force', Planck scale',' quantumgravity' and Hindu model of cosmic age in this paper the authors made an attempt to develop a unified model of spherical cosmology with very slow deceleration, angular velocity, temperature, redshift and large cosmic age.

\section{About cosmic rotation and quantum gravity}

\subsection{Cosmic rotation}

In our recently published paperthe authors proposed that, right from the beginning ofPlanck scale, universe is translating with light speed with a radius of $\mathrm{c} / \mathrm{H}$. If so, it is reasonable and natural to guess that, at every stage of cosmic expansion, for the expanding cosmic sphere, there exists certain angular velocity. By considering conservation of force, it is also reasonable to guess that, cosmic angular velocity is inversely proportional to cosmic size. With reference to Planck mass, at the beginning of comic evolution, angular velocity was very high and was equal to the Hubble parameter associated with Planck mass. Similarly for the current observable universe, angular velocity is equal to the current Hubble parameter. The main consequence of this proposal is that, righ from the beginning of cosmic evolution, universe rotates with light speed. Note that according to Michael Longo (Michael Longo 2011) the universe has a net angular momentum and was born in a spin. Whittaker says (E.T. Whittaker 1945):"however, that any of the mathematical-physical theories that have been put forward to explain spin (rotation) in the universe has yet won complete and universal acceptance; but progress has been so rapid in recent years, that it is reasonable to hope for a not long-delayed solution of this fundamental problem of cosmology". Yuri N. Obukhov (Yuri N. Obukhov 2000) says: "Whether our universe is rotating or not, it is of fundamental interest to understand the interrelation between rotation and other aspects of cosmological models as well as to understand the observational significance of an overall rotation".

\subsection{Quantum gravity}

In general, a unified branch of physics that connectsgeneral theory of relativity and quantum mechanics can be called as "quantum gravity". Clearly speaking, quantum gravity must show deep inner meaning at fundamental level for all possible energy scales. In this context, L.A. Glinka says - "Quantum gravity is one of the fundamental problems of modern theoretical physics. In spite of the significant efforts and various approaches, we are still very far of understanding the role of quantized gravitational fields in physical phenomena at high energies". To understand the advanced concepts of quantum gravity readers may refer L.A. Glinka's interesting paper (L. A. Glinka 2010). Note that Glinka's words clearly indicate the current uncertain status of quantum gravity. 'Quantum cosmology' isanother hot topic in current theoretical physics connected with the Planck scale and the expanding universe. Note that quantum cosmology attempts to explain those predictions related to the first phases of the early universe and also attempts to explain the current low energy scale observations of classical cosmology. For a full description of this new subject readers may refer the lecture notes by Martin Bojowald (Martin Bojowald 2011).

\section{Five unified, workable and simplified as- sumptions}

From the Planck scale to the scale of our observable universe and with reference to Hindu cosmic age, five workable and simple assumptions can be expressed as follows:

Assumption-1: Right from the beginning of Planck scale, universe is rotating with light speed from and about the cosmic center (But not from/about the Earth).

Comment-1: It may be noted that, without 'speed of light'there is no independent existence to Planck scale and without Planck scale there is no independent existence to physics and cosmology. In this paper, the authors are trying to give a heuristic cosmological significance to 'speed of light'.

Assumption-2: At any stage of cosmic evolution, ratio of Hubble parameter andangular velocity can be expressed as,

$$
\frac{H_{t}}{\omega_{t}} \cong\left\{1+\ln \left(\frac{\omega_{p l}}{\omega_{t}}\right)\right\} \cong \Upsilon_{t}
$$

Where $H_{t}$ is the Hubble parameter and $\omega_{p l}$ is the Planck scale angular velocity.

Comment-2: This assumption is new, ad-hoc and proposed with reference to the currently recommended magnitudes of Hubble parameter andCMBR temperature. Note that, in the earlier published paper, the authors assumed that, at any stage of cosmic expansion, Hubble parameter and cosmic angular velocity are equal in magnitude.It may be true that, ratio of angular velocity and Hubble parameter is model dependent. Interested readers may assume a different ratio of Hubble parameter and angular velocity and may try for fitting the current Hubble parameter and cosmic microwave back ground temperature.

Assumption-3: Right from the beginning of Planck scale, cosmic size follows the relation,

$$
R_{t} \cong \frac{G M_{t}}{c^{2}} \cong \frac{c}{\omega_{t}}
$$

Where $R_{t}, M_{t}$ and $\omega_{t}$ represent the radius, mass and angular velocity of the universe at time $t$ respectively.

Comment-3: This assumption is not new and can be seen in physics literature related with cosmology. With assumptions 1, 2 and 3 cosmic flatness and horizon problems can be understood. Now it is very simple to show that, at any stage of cosmic expansion, magnitude of centripetal force is the order of

$M_{t}\left(c^{2} / R_{t}\right) \cong M_{t} c \omega_{t} \cong\left(c^{4} / G\right)$.

Clearly speaking, at different stages of cosmic expansion,

$M_{1}\left(c^{2} / R_{1}\right) \cong M_{2}\left(c^{2} / R_{2}\right) \cong M_{3}\left(c^{2} / R_{3}\right) \cong\left(c^{4} / G\right)$.

Thinking in this way, at any stage of cosmic expansion, angular momentum can be shown to be

$L_{t} \approx M_{t} c R_{t} \approx\left(G M_{t}^{2} / c\right) \approx\left(M_{t} / M_{p l}\right)^{2} \hbar \approx\left(R_{t} / R_{p l}\right)^{2} \hbar$

Thus in this paper, the authors made an attempt to give priority to "constant centripetal force" or "conservation of centripetal force" rather than "conservation of angular momentum".

Assumption-4: Right from the beginning of Planck scale, at any stage of cosmic expansion, cosmic gravitational potential energy and total thermal energy are equal in magnitude and can be expressed asfollows. 


$$
\frac{3}{5} \frac{G M_{t}^{2}}{R_{t}} \cong a T_{t}^{4}\left[\frac{4 \pi}{3}\left(R_{t}^{3}\right)\right]
$$

Comment-4: This assumption is new and can be given some consideration for in depth analysis with respect to energy conservation in the expanding universe.

Assumption-5: At any stage of cosmic expansion, 'light speed' play a key role in the following way.

$$
\left.\begin{array}{l}
R_{t} \cong \sqrt{(c t)\left(c / H_{t}\right)} \cong c \sqrt{t / H_{t}} \\
\rightarrow R_{t} / c \cong 1 / \omega_{t} \cong \sqrt{t / H_{t}} \text { and } \\
t \cong \frac{H_{t}}{\omega_{t}^{2}} \cong\left[1+\ln \left(\frac{\omega_{p l}}{\omega t}\right)\right]\left(\frac{1}{\omega_{t}}\right)
\end{array}\right\}
$$

Where $t$ is the cosmic age, $H_{t}$ is the Hubble parameter and $\omega_{t}$ is the angular velocity.

Comment-5: With this assumption, Hindu's model of cosmic age can be fitted with a factor of $1 / 2$. another interesting point is that, at any stage of cosmic expansion, expansion speed can be expressed as

$$
v_{t} \cong \frac{R_{t}}{t} \cong\left\{1+\ln \left(\frac{\omega_{p l}}{\omega_{t}}\right)\right\}^{-1} c \cong\left(\frac{\omega_{t}}{H_{t}}\right) c
$$

And seems to be decreasing very slowly by the factor

$$
\left\{1+\ln \left(\frac{\omega_{p l}}{\omega_{t}}\right)\right\}
$$

At present, it seems that

$$
v_{0} \cong \frac{R_{0}}{t_{0}} \cong\left\{1+\ln \left(\frac{\omega_{p l}}{\omega_{0}}\right)\right\}^{-1} c \cong c / 146
$$

Hence it ispossible to say that, at present, universe is decelerating with a velocity of $v_{0} \cong c / 146$ and rate of deceleration is very small and seems to be beyond the scope of current observations. See table 1. Data of table-1 seems to support the recent Super novae observational conclusions of uniform rate of expansion. This proposal can also be conformed from the isotropic nature of current CMBR temperature.

\section{To connect the cosmic physicalparameters}

Following these assumptions, Planck scale Hubble parameter and angular velocity both can be assumed to be equal in magnitude and can be expressed as follows.

$$
\begin{aligned}
& H_{p l} \cong \omega_{p l} \cong \frac{c^{3}}{G M_{p l}} \cong \frac{c}{R_{p l}} \\
& \cong 1.85492 \times 10^{43}{\mathrm{rad} . \mathrm{sec}^{-1}}^{-1}
\end{aligned}
$$

Where $R_{p l} \cong G M_{p l} / c^{2} \cong \sqrt{G \hbar / c^{3}} \cong 1.6162 \times 10^{-35} \mathrm{~m}$

Is the assumed radius connected with Planck mass.

Planck scale temperature can be expressed as

$$
\begin{aligned}
& T_{p l} \cong\left(\frac{9 \omega_{p l}^{2} c^{2}}{20 \pi G a}\right)^{\frac{1}{4}} \cong\left(\frac{9 H_{p l}^{2} c^{2}}{20 \pi G a}\right)^{\frac{1}{4}} \\
& \cong 9.67792 \times 10^{31} \mathrm{~K}
\end{aligned}
$$

At any stage of cosmic expansion, if cosmic temperature is known,

Step-1: Angular velocity can be estimated with the following relation.

$$
a T_{t}^{4} \cong \frac{9 c^{2} \omega_{t}^{2}}{20 \pi G} \text { and } \omega_{t} \cong \sqrt{\frac{20 \pi G a T_{t}^{4}}{9 c^{2}}}
$$

Step-2: Hubble parameter can be estimated with the following relation.

$$
H_{t} \cong \omega_{t}\left\{1+\ln \left(\frac{\omega_{p l}}{\omega_{t}}\right)\right\}
$$

It is having the following key applications in cosmology.

1) Current CMBR temperature can be fitted approximately.

2) A very simple relation for CMBR redshift can be developed. See section-8.

3) Standard cosmology's predicted redshift of 1100 connected with recombination temperature of $3000 \mathrm{~K}$ can be fitted very easily. See section-8.

4) At every stage of expansion, qualitatively Hawking's 'black hole temperature formula' like relation can be obtained. See relation (9).

5) General relativity, Quantum mechanics, Planck scale high temperatures, current \& future low temperatures can be studied in a unified manner and a unified model of scale independent quantum gravity/cosmologycan be developed at fundamental level.

With reference to Planck mass and by splitting the radiation constant, if cosmic angular velocity is known, cosmic temperature can be estimated with the following relation.

$$
\begin{aligned}
T_{t} & \cong\left(\frac{9 \omega_{t}^{2} c^{2}}{20 \pi G a}\right)^{1 / 4} \cong\left(\frac{135}{20 \pi^{3}}\right)^{1 / 4}\left(\frac{\hbar c^{3}}{k_{B} G \sqrt{M_{t} M_{p l}}}\right) \\
& \cong\left(\frac{135}{20 \pi^{3}}\right)^{1 / 4}\left(\frac{M_{t}}{M_{p l}}\right)^{1 / 2}\left(\frac{\hbar c^{3}}{k_{B} G M_{t}}\right) .
\end{aligned}
$$

In this relation, the expression $\left(\frac{\hbar c^{3}}{k_{B} G M_{t}}\right)$ qualitatively can be compared with the famous Hawking's Black hole temperature formula (Hawking, S.W 1975). Considering this relation, quantum mechanics, general theory of relativity and Planck scale can be studied in a unified manner and quantum cosmology can be put into main stream cosmological observations.

\section{The characteristic equations of current uni- verse in this unified model of cosmology}

As per the 2015 Planck data (P.A.R. Ade et al, Fixsen, D.J. 2009) the current value of the Hubble parameter is reported to be:

Planck TT+low P: $(67.31 \pm 0.96) \mathrm{km} / \mathrm{sec} / \mathrm{Mpc}$;

Planck TE+low P: $(67.73 \pm 0.92) \mathrm{km} / \mathrm{sec} / \mathrm{Mpc}$;

Planck TT,TE,EE+low P: $(67.7 \pm 0.66) \mathrm{km} / \mathrm{sec} / \mathrm{Mpc}$;

As per the 2015 Planck data, the current $T_{0}$ is :

Planck TT + lowP + BAO: $(2.722 \pm 0.027) \mathrm{K}$,

Planck TT; TE; EE + low P + BAO: $(2.718 \pm 0.021) \mathrm{K}$

and upper limit seesms to be $2.722+0.027 \cong 2.749 \mathrm{~K}$.

Step-1: With reference to the upper limit of recommended current angular velocity can be estimated as follows: 
$\omega_{0} \cong \sqrt{\frac{20 \pi G a T_{0}^{4}}{9 c^{2}}} \cong 1.496613 \times 10^{-20} \mathrm{rad} / \mathrm{sec}$

Step-2: Current Hubble parameter can be estimated as follows:

$$
\begin{aligned}
& H_{0} \cong \omega_{0}\left\{1+\ln \left(\frac{\omega_{p l}}{\omega_{0}}\right)\right\} \cong 2.18921 \times 10^{-18} \mathrm{sec}^{-1} \\
& \cong 67.5524 \mathrm{~km} / \mathrm{sec} / \mathrm{Mpc}
\end{aligned}
$$

Current cosmic mass and radius can be estimated as,

$$
\left.\begin{array}{c}
M_{0} \cong \frac{R_{0} c^{2}}{G} \cong \frac{c^{3}}{G \omega_{0}} \cong 2.6976 \times 10^{55} \mathrm{~kg} \\
R_{0} \cong \frac{G M_{0}}{c^{2}} \cong \frac{c}{\omega_{0}} \cong 2.00 \times 10^{28} \mathrm{~m}
\end{array}\right\}
$$

The two impossible things in cosmology are: 1) Measuring the cosmic size.2) Measuring the cosmic mass. It may be noted that, with reference to current Hubble radius, $~ 68 \%$ dark energy and $\sim 32 \%$ (observable matter and dark matter) total estimated mass of current universe is $2.48 \times 10^{54} \mathrm{~kg}$. This can be compared with the proposed estimate of $2.70 \times 10^{55} \mathrm{~kg}$. Estimation of observable cosmic mass mainly depends on "counting the number of "weighing the central core mass of all the galaxies', 'counting the number of stars in all of the galaxies' and 'weighing the individual mass of stars' etc. This entire procedure ismainly based on 'observational approach' and needs so many correction factors. Two interesting points are: 1) Day by day, 'cosmic acceleration' and 'dark energy' both are losing their identity. 2) Modified Newtonian dynamics (MOND) taking a leading role in understanding the galactic rotational curves (Milgrom, M. 1983, J. R. Brownstein and J. W. Moffat 2006, Edmund A. Chadwick et al 2013) and day by day, dark matter also losing its identity. Hence in future it may be easy to estimate the cosmic mass. With future cosmological observa- tions and other models of cosmology, these proposed magnitudes of cosmic mass and size can be considered as the characteristic limiting magnitudes.

\section{Cosmic age}

In general, cosmic age is 'model dependent' and 'cosmic size dependent'. In this proposed model, cosmic age estimation is very simple and direct. With assumption-5 and from the beginning of Planck scale, cosmic age can be estimated as follows.

$$
t \cong \frac{R_{t}^{2} H_{t}}{c^{2}} \cong \frac{H_{t}}{\omega_{t}^{2}} \cong\left[1+\ln \left(\frac{\omega_{p l}}{\omega_{t}}\right)\right]\left(\frac{1}{\omega_{t}}\right)
$$

For the current case,

$$
t_{0} \cong \frac{H_{0}}{\omega_{0}^{2}} \cong\left[1+\ln \left(\frac{\omega_{p l}}{\omega_{0}}\right)\right]\left(\frac{1}{\omega_{0}}\right)
$$

$$
\cong 9.774 \times 10^{21} \mathrm{sec} \cong 309.72 \text { TrillionYears }
$$

This estimated time is matching with the Hindu model of cosmic agewith a factor of $1 / 2$ and needs further study. Note that, according to Hindu cosmology, total age of the universe is 311.4 trillion years and current cosmic age is just greater than 155.7 trillion years. From relation (12) estimated current cosmic radiusis146 times higher than the current Hubble radius and current cosmic

\begin{tabular}{|c|c|c|c|c|c|c|}
\hline $\begin{array}{c}\text { Assumed angular } \\
\text { velocity } \\
(\mathrm{rad} / \mathrm{sec})\end{array}$ & $\begin{array}{c}\text { Estimated Hubble } \\
\text { parameter } \\
(1 / \mathrm{sec})\end{array}$ & $\begin{array}{l}\text { Estimated cosmic } \\
\text { radius } \\
(\mathrm{m})\end{array}$ & $\begin{array}{c}\text { Estimated cosmic } \\
\text { mass } \\
(\mathrm{kg})\end{array}$ & $\begin{array}{c}\text { Estimated cosmic } \\
\text { temperature } \\
\text { (T) }\end{array}$ & $\begin{array}{c}\text { Estimated cosmic } \\
\text { age } \\
(\mathrm{sec})\end{array}$ & $\begin{array}{l}\text { Estimated cosmic } \\
\text { rate of expansion } \\
(\mathrm{m} / \mathrm{sec})\end{array}$ \\
\hline $1.49229 \mathrm{E}+42$ & $5.25304 \mathrm{E}+42$ & $2.00894 \mathrm{E}-34$ & $2.70540 \mathrm{E}-07$ & $2.74502 \mathrm{E}+31$ & $2.35886 \mathrm{E}-42$ & $8.51656 \mathrm{E}+07$ \\
\hline $1.49229 \mathrm{E}+41$ & $8.68918 \mathrm{E}+41$ & $2.00894 \mathrm{E}-33$ & $2.70540 \mathrm{E}-06$ & $8.68052 \mathrm{E}+30$ & 3.90184E-41 & $5.14869 \mathrm{E}+07$ \\
\hline $1.49229 \mathrm{E}+40$ & $1.21253 \mathrm{E}+41$ & $2.00894 \mathrm{E}-32$ & $2.70540 \mathrm{E}-05$ & $2.74502 \mathrm{E}+30$ & 5.44483E-40 & $3.68962 \mathrm{E}+07$ \\
\hline 1.49229E+39 & $1.55614 \mathrm{E}+40$ & 2.00894E-31 & 2.70540E-04 & $8.68052 \mathrm{E}+29$ & $6.98781 \mathrm{E}-39$ & $2.87492 \mathrm{E}+07$ \\
\hline $1.49229 \mathrm{E}+38$ & $1.89976 \mathrm{E}+39$ & $2.00894 \mathrm{E}-30$ & $2.70540 \mathrm{E}-03$ & $2.74502 \mathrm{E}+29$ & 8.53079E-38 & $2.35492 \mathrm{E}+07$ \\
\hline $1.49229 \mathrm{E}+37$ & $2.24337 \mathrm{E}+38$ & $2.00894 \mathrm{E}-29$ & $2.70540 \mathrm{E}-02$ & $8.68052 \mathrm{E}+28$ & $1.00738 \mathrm{E}-36$ & $1.99422 \mathrm{E}+07$ \\
\hline $1.49229 \mathrm{E}+35$ & $2.93060 \mathrm{E}+36$ & $2.00894 \mathrm{E}-27$ & $2.70540 \mathrm{E}+00$ & $8.68052 \mathrm{E}+27$ & $1.31597 \mathrm{E}-34$ & $1.52658 \mathrm{E}+07$ \\
\hline $1.49229 \mathrm{E}+34$ & $3.27421 \mathrm{E}+35$ & $2.00894 \mathrm{E}-26$ & $2.70540 \mathrm{E}+01$ & $2.74502 \mathrm{E}+27$ & $1.47027 \mathrm{E}-33$ & $1.36637 \mathrm{E}+07$ \\
\hline $1.49229 \mathrm{E}+33$ & $3.61782 \mathrm{E}+34$ & $2.00894 \mathrm{E}-25$ & $2.70540 \mathrm{E}+02$ & $8.68052 \mathrm{E}+26$ & $1.62457 \mathrm{E}-32$ & $1.23660 \mathrm{E}+07$ \\
\hline $1.49229 \mathrm{E}+32$ & $3.96144 \mathrm{E}+33$ & 2.00894E-24 & $2.70540 \mathrm{E}+03$ & $2.74502 \mathrm{E}+26$ & $1.77887 \mathrm{E}-31$ & $1.12933 \mathrm{E}+07$ \\
\hline $1.49229 \mathrm{E}+31$ & $4.30505 E+32$ & $2.00894 \mathrm{E}-23$ & $2.70540 \mathrm{E}+04$ & $8.68052 \mathrm{E}+25$ & $1.93317 \mathrm{E}-30$ & $1.03919 \mathrm{E}+07$ \\
\hline $1.49229 \mathrm{E}+30$ & $4.64867 \mathrm{E}+31$ & $2.00894 \mathrm{E}-22$ & $2.70540 \mathrm{E}+05$ & $2.74502 \mathrm{E}+25$ & $2.08747 \mathrm{E}-29$ & $9.62381 \mathrm{E}+06$ \\
\hline $1.49229 \mathrm{E}+29$ & $4.99228 \mathrm{E}+30$ & $2.00894 \mathrm{E}-21$ & $2.70540 \mathrm{E}+06$ & $8.68052 \mathrm{E}+24$ & $2.24176 \mathrm{E}-28$ & $8.96141 \mathrm{E}+06$ \\
\hline $1.49229 \mathrm{E}+28$ & $5.33589 \mathrm{E}+29$ & 2.00894E-20 & $2.70540 \mathrm{E}+07$ & $2.74502 \mathrm{E}+24$ & $2.39606 \mathrm{E}-27$ & $8.38432 \mathrm{E}+06$ \\
\hline $1.49229 \mathrm{E}+26$ & $6.02312 \mathrm{E}+27$ & 2.00894E-18 & $2.70540 \mathrm{E}+09$ & $2.74502 \mathrm{E}+23$ & $2.70466 \mathrm{E}-25$ & $7.42769 \mathrm{E}+06$ \\
\hline $1.49229 \mathrm{E}+25$ & $6.36673 E+26$ & 2.00894E-17 & $2.70540 \mathrm{E}+10$ & $8.68052 \mathrm{E}+22$ & $2.85896 \mathrm{E}-24$ & $7.02682 \mathrm{E}+06$ \\
\hline $1.49229 \mathrm{E}+24$ & $6.71035 \mathrm{E}+25$ & $2.00894 \mathrm{E}-16$ & $2.70540 \mathrm{E}+11$ & $2.74502 \mathrm{E}+22$ & $3.01326 \mathrm{E}-23$ & $6.66700 \mathrm{E}+06$ \\
\hline $1.49229 \mathrm{E}+23$ & $7.05396 \mathrm{E}+24$ & $2.00894 \mathrm{E}-15$ & $2.70540 \mathrm{E}+12$ & $8.68052 \mathrm{E}+21$ & $3.16755 \mathrm{E}-22$ & $6.34223 \mathrm{E}+06$ \\
\hline $1.49229 \mathrm{E}+22$ & $7.39757 \mathrm{E}+23$ & $2.00894 \mathrm{E}-14$ & $2.70540 \mathrm{E}+13$ & $2.74502 \mathrm{E}+21$ & $3.32185 \mathrm{E}-21$ & $6.04764 \mathrm{E}+06$ \\
\hline $1.49229 \mathrm{E}+21$ & 7.74119E+22 & $2.00894 \mathrm{E}-13$ & $2.70540 \mathrm{E}+14$ & $8.68052 \mathrm{E}+20$ & $3.47615 \mathrm{E}-20$ & $5.77920 \mathrm{E}+06$ \\
\hline $1.49229 \mathrm{E}+20$ & $8.08480 \mathrm{E}+21$ & $2.00894 \mathrm{E}-12$ & $2.70540 \mathrm{E}+15$ & $2.74502 \mathrm{E}+20$ & $3.63045 \mathrm{E}-19$ & $5.53358 \mathrm{E}+06$ \\
\hline $1.49229 \mathrm{E}+19$ & $8.42841 \mathrm{E}+20$ & $2.00894 \mathrm{E}-11$ & $2.70540 \mathrm{E}+16$ & $8.68052 \mathrm{E}+19$ & $3.78475 \mathrm{E}-18$ & $5.30798 \mathrm{E}+06$ \\
\hline $1.49229 \mathrm{E}+18$ & $8.77203 E+19$ & $2.00894 \mathrm{E}-10$ & $2.70540 \mathrm{E}+17$ & $2.74502 \mathrm{E}+19$ & 3.93905E-17 & $5.10006 \mathrm{E}+06$ \\
\hline $1.49229 \mathrm{E}+17$ & $9.11564 \mathrm{E}+18$ & 2.00894E-09 & $2.70540 \mathrm{E}+18$ & $8.68052 \mathrm{E}+18$ & $4.09334 \mathrm{E}-16$ & $4.90781 \mathrm{E}+06$ \\
\hline $1.49229 \mathrm{E}+16$ & $9.45925 \mathrm{E}+17$ & 2.00894E-08 & $2.70540 \mathrm{E}+19$ & $2.74502 \mathrm{E}+18$ & 4.24764E-15 & $4.72953 \mathrm{E}+06$ \\
\hline $1.49229 \mathrm{E}+15$ & $9.80287 \mathrm{E}+16$ & 2.00894E-07 & $2.70540 \mathrm{E}+20$ & $8.68052 \mathrm{E}+17$ & 4.40194E-14 & $4.56375 \mathrm{E}+06$ \\
\hline $1.49229 \mathrm{E}+14$ & $1.01465 \mathrm{E}+16$ & 2.00894E-06 & $2.70540 \mathrm{E}+21$ & $2.74502 \mathrm{E}+17$ & $4.55624 \mathrm{E}-13$ & $4.40920 \mathrm{E}+06$ \\
\hline $1.49229 \mathrm{E}+13$ & $1.04901 \mathrm{E}+15$ & $2.00894 \mathrm{E}-05$ & $2.70540 \mathrm{E}+22$ & $8.68052 \mathrm{E}+16$ & 4.71054E-12 & $4.26477 \mathrm{E}+06$ \\
\hline $1.49229 \mathrm{E}+12$ & $1.08337 \mathrm{E}+14$ & $2.00894 \mathrm{E}-04$ & $2.70540 \mathrm{E}+23$ & $2.74502 \mathrm{E}+16$ & 4.86484E-11 & $4.12951 \mathrm{E}+06$ \\
\hline $1.49229 \mathrm{E}+11$ & $1.11773 \mathrm{E}+13$ & 2.00894E-03 & $2.70540 \mathrm{E}+24$ & $8.68052 \mathrm{E}+15$ & $5.01913 \mathrm{E}-10$ & $4.00256 \mathrm{E}+06$ \\
\hline $1.49229 \mathrm{E}+10$ & $1.15209 \mathrm{E}+12$ & $2.00894 \mathrm{E}-02$ & $2.70540 \mathrm{E}+25$ & $2.74502 \mathrm{E}+15$ & 5.17343E-09 & $3.88318 \mathrm{E}+06$ \\
\hline
\end{tabular}
age is $1462=21397$ times higher than the currently believed cosmic age of 13.8 billion years. No that, in this model, assumed cosmic time is a function of cosmic angular velocity and is subjected to current and future observational estimations of magnitude of cosmic angular velocity.

Table 1:To Estiamte Various Parameters of the Expanding and Rotating Universe 


\begin{tabular}{|c|c|c|c|c|c|c|}
\hline $1.49229 \mathrm{E}+09$ & $1.18645 \mathrm{E}+11$ & $2.00894 \mathrm{E}-01$ & $2.70540 \mathrm{E}+26$ & $8.68052 \mathrm{E}+14$ & $5.32773 \mathrm{E}-08$ & $3.77072 \mathrm{E}+06$ \\
\hline $1.49229 \mathrm{E}+08$ & $1.22082 \mathrm{E}+10$ & $2.00894 \mathrm{E}+00$ & $2.70540 \mathrm{E}+27$ & $2.74502 \mathrm{E}+14$ & 5.48203E-07 & $3.66459 \mathrm{E}+06$ \\
\hline 1.49229E+07 & $1.25518 \mathrm{E}+09$ & $2.00894 \mathrm{E}+01$ & $2.70540 \mathrm{E}+28$ & $8.68052 \mathrm{E}+13$ & 5.63633E-06 & $3.56427 \mathrm{E}+06$ \\
\hline $1.49229 \mathrm{E}+06$ & $1.28954 \mathrm{E}+08$ & $2.00894 \mathrm{E}+02$ & $2.70540 \mathrm{E}+29$ & $2.74502 \mathrm{E}+13$ & 5.79063E-05 & $3.46929 \mathrm{E}+06$ \\
\hline $1.49229 \mathrm{E}+05$ & $1.32390 \mathrm{E}+07$ & $2.00894 \mathrm{E}+03$ & $2.70540 \mathrm{E}+30$ & $8.68052 \mathrm{E}+12$ & 5.94492E-04 & $3.37925 \mathrm{E}+06$ \\
\hline $1.49229 \mathrm{E}+04$ & $1.35826 \mathrm{E}+06$ & $2.00894 \mathrm{E}+04$ & $2.70540 \mathrm{E}+31$ & $2.74502 \mathrm{E}+12$ & 6.09922E-03 & $3.29376 \mathrm{E}+06$ \\
\hline $1.49229 \mathrm{E}+03$ & $1.39262 \mathrm{E}+05$ & $2.00894 \mathrm{E}+05$ & $2.70540 \mathrm{E}+32$ & $8.68052 \mathrm{E}+11$ & $6.25352 \mathrm{E}-02$ & $3.21249 \mathrm{E}+06$ \\
\hline 1.49229E+02 & $1.42698 \mathrm{E}+04$ & $2.00894 \mathrm{E}+06$ & $2.70540 \mathrm{E}+33$ & $2.74502 \mathrm{E}+11$ & $6.40782 \mathrm{E}-01$ & $3.13513 \mathrm{E}+06$ \\
\hline $1.49229 \mathrm{E}+01$ & $1.46135 \mathrm{E}+03$ & $2.00894 \mathrm{E}+07$ & $2.70540 \mathrm{E}+34$ & $8.68052 \mathrm{E}+10$ & $6.56212 \mathrm{E}+00$ & $3.06142 \mathrm{E}+06$ \\
\hline $1.49229 \mathrm{E}+00$ & $1.49571 \mathrm{E}+02$ & $2.00894 \mathrm{E}+08$ & $2.70540 \mathrm{E}+35$ & $2.74502 \mathrm{E}+10$ & $6.71642 \mathrm{E}+01$ & $2.99108 \mathrm{E}+06$ \\
\hline $1.49229 \mathrm{E}-01$ & $1.53007 \mathrm{E}+01$ & $2.00894 \mathrm{E}+09$ & $2.70540 \mathrm{E}+36$ & $8.68052 \mathrm{E}+09$ & $6.87072 \mathrm{E}+02$ & $2.92391 \mathrm{E}+06$ \\
\hline 1.49229E-02 & $1.56443 \mathrm{E}+00$ & $2.00894 \mathrm{E}+10$ & $2.70540 \mathrm{E}+37$ & $2.74502 \mathrm{E}+09$ & $7.02501 \mathrm{E}+03$ & $2.85969 \mathrm{E}+06$ \\
\hline 1.49229E-03 & $1.59879 \mathrm{E}-01$ & $2.00894 \mathrm{E}+11$ & $2.70540 \mathrm{E}+38$ & $8.68052 \mathrm{E}+08$ & $7.17931 \mathrm{E}+04$ & $2.79823 \mathrm{E}+06$ \\
\hline $1.49229 \mathrm{E}-04$ & $1.63315 \mathrm{E}-02$ & $2.00894 \mathrm{E}+12$ & $2.70540 \mathrm{E}+39$ & $2.74502 \mathrm{E}+08$ & $7.33361 \mathrm{E}+05$ & $2.73936 \mathrm{E}+06$ \\
\hline $1.49229 \mathrm{E}-05$ & $1.66751 \mathrm{E}-03$ & $2.00894 \mathrm{E}+13$ & $2.70540 \mathrm{E}+40$ & $8.68052 \mathrm{E}+07$ & $7.48791 \mathrm{E}+06$ & $2.68291 \mathrm{E}+06$ \\
\hline $1.49229 \mathrm{E}-06$ & $1.70187 \mathrm{E}-04$ & $2.00894 \mathrm{E}+14$ & $2.70540 \mathrm{E}+41$ & $2.74502 \mathrm{E}+07$ & $7.64221 \mathrm{E}+07$ & $2.62874 \mathrm{E}+06$ \\
\hline $1.49229 \mathrm{E}-07$ & $1.73624 \mathrm{E}-05$ & $2.00894 \mathrm{E}+15$ & $2.70540 \mathrm{E}+42$ & $8.68052 \mathrm{E}+06$ & $7.79651 \mathrm{E}+08$ & $2.57671 \mathrm{E}+06$ \\
\hline $1.49229 \mathrm{E}-08$ & $1.77060 \mathrm{E}-06$ & $2.00894 \mathrm{E}+16$ & $2.70540 \mathrm{E}+43$ & $2.74502 \mathrm{E}+06$ & $7.95080 \mathrm{E}+09$ & $2.52671 \mathrm{E}+06$ \\
\hline 1.49229E-09 & $1.80496 \mathrm{E}-07$ & $2.00894 \mathrm{E}+17$ & $2.70540 \mathrm{E}+44$ & $8.68052 \mathrm{E}+05$ & $8.10510 \mathrm{E}+10$ & $2.47861 \mathrm{E}+06$ \\
\hline $1.49229 \mathrm{E}-10$ & 1.83932E-08 & $2.00894 \mathrm{E}+18$ & $2.70540 \mathrm{E}+45$ & $2.74502 \mathrm{E}+05$ & $8.25940 \mathrm{E}+11$ & $2.43230 \mathrm{E}+06$ \\
\hline $1.49229 \mathrm{E}-11$ & 1.87368E-09 & $2.00894 \mathrm{E}+19$ & $2.70540 \mathrm{E}+46$ & $8.68052 \mathrm{E}+04$ & $8.41370 \mathrm{E}+12$ & $2.38770 \mathrm{E}+06$ \\
\hline $1.49229 \mathrm{E}-12$ & $1.90804 \mathrm{E}-10$ & $2.00894 \mathrm{E}+20$ & $2.70540 \mathrm{E}+47$ & $2.74502 \mathrm{E}+04$ & $8.56800 \mathrm{E}+13$ & $2.34470 \mathrm{E}+06$ \\
\hline $1.49229 \mathrm{E}-13$ & $1.94240 \mathrm{E}-11$ & $2.00894 \mathrm{E}+21$ & $2.70540 \mathrm{E}+48$ & $8.68052 \mathrm{E}+03$ & $8.72230 \mathrm{E}+14$ & $2.30322 \mathrm{E}+06$ \\
\hline $1.49229 \mathrm{E}-14$ & $1.97677 \mathrm{E}-12$ & $2.00894 \mathrm{E}+22$ & $2.70540 \mathrm{E}+49$ & $2.74502 \mathrm{E}+03$ & $8.87659 \mathrm{E}+15$ & $2.26318 \mathrm{E}+06$ \\
\hline $1.49229 \mathrm{E}-15$ & $2.01113 \mathrm{E}-13$ & $2.00894 \mathrm{E}+23$ & $2.70540 \mathrm{E}+50$ & $8.68052 \mathrm{E}+02$ & $9.03089 \mathrm{E}+16$ & $2.22452 \mathrm{E}+06$ \\
\hline $1.49229 \mathrm{E}-16$ & $2.04549 \mathrm{E}-14$ & $2.00894 \mathrm{E}+24$ & $2.70540 \mathrm{E}+51$ & $2.74502 \mathrm{E}+02$ & $9.18519 \mathrm{E}+17$ & $2.18715 \mathrm{E}+06$ \\
\hline $1.49229 \mathrm{E}-17$ & $2.07985 \mathrm{E}-15$ & $2.00894 \mathrm{E}+25$ & $2.70540 \mathrm{E}+52$ & $8.68052 \mathrm{E}+01$ & $9.33949 \mathrm{E}+18$ & $2.15101 \mathrm{E}+06$ \\
\hline $1.49229 \mathrm{E}-18$ & $2.11421 \mathrm{E}-16$ & $2.00894 \mathrm{E}+26$ & $2.70540 \mathrm{E}+53$ & $2.74502 \mathrm{E}+01$ & $9.49379 \mathrm{E}+19$ & $2.11605 \mathrm{E}+06$ \\
\hline $1.49229 \mathrm{E}-19$ & $2.14857 \mathrm{E}-17$ & $2.00894 \mathrm{E}+27$ & $2.70540 \mathrm{E}+54$ & $8.68052 \mathrm{E}+00$ & $9.64809 \mathrm{E}+20$ & $2.08221 \mathrm{E}+06$ \\
\hline $1.49229 \mathrm{E}-20$ & 2.18293E-18 & $2.00894 \mathrm{E}+28$ & $2.70540 \mathrm{E}+55$ & $2.74502 \mathrm{E}+00$ & $9.80238 \mathrm{E}+21$ & $2.04944 \mathrm{E}+06$ \\
\hline
\end{tabular}

\section{Practical applications of current angular- velocity in this unified model}

\section{a) Galactic revolving speed:}

For the current light speed rotating cosmic model, on the equatorial plane, galactic revolving speed can be expressed as,

$$
\left(v_{g}\right)_{r e v} \cong r_{g} \omega_{0} \leq c
$$

Here, $r_{g}$ and $\left(v_{g}\right)_{r v v}$ represent the galactic distance from the cosmic center and galacticrevolving speed corresponding to the cosmic angular velocity, respectively. The important point to be noted is that, even though $\frac{\left(v_{g}\right)_{r e v}}{c}$ is always less than 1 , the proposed velocity refers to galactic "revolution speed" about the cosmic center and the proposed distance refers to galaxy distance from the cosmic center. Importantly, actual galactic "revolving speeds" have never been confirmed by any direct cosmological observations. This is for further study.

b) Galactic receding speed:

In modeling the current expanding universe, on the equatorial plane, galactic receding speed can be expressed as follows.

$$
\left(v_{g}\right)_{\text {rec }} \cong\left(\frac{r_{g}}{R_{0}}\right) v_{0} \leq v_{0}
$$

In Hubble's law (Hubble, E.P. 1929, 1947), velocity refers to galactic "receding speed" and distance refers to "distance between galaxy and observer." Thus Hubble's law appears to be a natural physical consequence in this model.

c) Galactic centripetal acceleration:

1) For any revolving galaxy, galactic centripetal acceleration can be expressed as:

$a_{g} \cong \omega_{0}\left(v_{g}\right)_{r e v} \cong r_{g} \omega_{0}^{2}$

2) For any satellite that is assumed to be revolving at a distance

$r_{\text {satelite }}$ from the cosmic center, centripetal acceleration can be expressed as: $a_{\text {satellite }} \cong \omega_{0}\left(v_{g}\right)_{\text {rev }} \cong r_{\text {satellite }} \omega_{0}^{2}$

Based on the above applications, and by measuring actual galactic "revolving speeds", the current cosmic centripetal acceleration can be estimated.

\section{d) Galactic rotational curves:}

The current dominant paradigm is that galaxies are embedded in halos of cold dark matter (CDM), made of non-baryonic weaklyinteracting massive particles. However, an alternative way to explain the observed rotation curves of galaxies is the postulate that, for gravitational accelerations below a certain value $a_{0} \cong(1.2 \pm 0.3) \times 10^{-10} \mathrm{~m} \cdot \mathrm{sec}^{-2}$, The true gravitational field strength $g$ approaches $\sqrt{g_{N} g}$, where $g_{N}$ is the usual Newtonian gravitational field strength (as calculated from the observed distribution of visible matter). This paradigm is known as modified Newtonian dynamics (MOND). Here, $a_{0} \cong(1.2 \pm 0.3) \times 10^{-10} \mathrm{~m} \cdot \mathrm{sec}^{-2}$. In the light speed rotating cosmic model, by considering the galactic revolving speed $\left(v_{g}\right)_{r e v}$ about the cosmic center, the magnitude of galactic centripetal acceleration can be assumed to vary as:

$a_{g} \cong \omega_{0}\left(v_{g}\right)_{r e v} \cong r_{g} \omega_{0}^{2}$

Where $r_{g}$ is the distance between galaxy and the cosmic center. Now rotational speed of a star in any galaxy can be represented as follows:

$$
\begin{aligned}
\left(v_{\text {star }}\right)_{\text {rev }} & \propto \sqrt[4]{G M_{g} \omega_{0}\left(v_{g}\right)_{r e v}} \\
& \propto \sqrt[4]{G M_{g} r_{g} \omega_{0}^{2}}
\end{aligned}
$$

$M_{g}$ is the mass of the galaxy. With an assumed universal proportionality ratio of 1 , and by knowing the galactic mass and actual revolving speeds of galactic stars, galactic revolving speed and galactic distance from the cosmic center can be approximated in the following way: 


$$
\left.\begin{array}{l}
\left(v_{g}\right)_{r e v} \cong \frac{\left(v_{s t a r}\right)_{r e v}^{4}}{G M_{g} \omega_{0}} \text { and } \\
r_{g} \cong \frac{\left(v_{g}\right)_{r e v}}{\omega_{0}} \cong \frac{\left(v_{s t a r}\right)_{r v v}^{4}}{G M_{g} \omega_{0}^{2}}
\end{array}\right\}
$$

By knowing our mother galactic mass and rotational curves, our galactic distance from the cosmic center can be approximated. By considering the different model-dependent proportionality ratios and correlating all of the data, finally the correct magnitude of the proportionality ratio can be fitted. This is for further study.

\section{Model equation of cosmic non-linear red- shift and to estimate the cosmic angular ve- locity}

In this section, in a semi-empirical approach, the authors propose a very simple model equation for observed and predicted cosmic redshifts, including galactic and CMBR redshifts. These are for further research and analysis. With reference to the proposed assumptions,

$$
\left\{1+\ln \left(\frac{\omega_{p l}}{\omega_{0}}\right)\right\} \cong\left\{1+\ln \left(\frac{R_{t}}{R_{p l}}\right)\right\}
$$

Thus at any stage of cosmic expansion in the past,

$$
\left\{1+\ln \left(\frac{\omega_{p l}}{\omega_{t}}\right)\right\} \cong\left\{1+\ln \left(\frac{R_{t}}{R_{p l}}\right)\right\}
$$

Based on this relation, one particularly simple model equation under current study is:

$$
\begin{aligned}
& Z \cong \sqrt{\frac{\omega_{t}}{\omega_{0}}-1} \cong \sqrt{\frac{R_{0}}{R_{t}}-1} \cong \sqrt{\frac{G M_{0}}{c^{2} R_{t}}-1} \\
& \text { where } \omega_{t}>\omega_{0}, R_{t}<R_{0}, \text { and } M_{0} \cong c^{3} / G \omega_{0} .
\end{aligned}
$$

Where $\omega_{0}$ and $\omega_{t}$ represent current and past cosmic angular velocity respectively. Similarly $R_{0}$ and $R_{t}$ represent current and past decreasing cosmic radii, respectively.Thus in this model, by knowing or guessing the galactic redshift, cosmic angular velocity can be estimated.

With reference to cosmic center and by following relation (23) and Minkowski's relativistic Doppler shift formula, galactic redshift (connected with simultaneous cosmic expansion speed and light speed rotation) may be considered for further study and analysis.

With reference to the proposed assumptions, relation (24) can be obtained in the following semi-empirical approach. Let,

$$
\begin{aligned}
& {\left[1+\ln \left(\frac{R_{0}}{R_{p l}}\right)\right] \cong \Upsilon_{0} \text { and }\left[1+\ln \left(\frac{R_{t}}{R_{p l}}\right)\right] \cong \Upsilon_{t}} \\
& Z \cong \sqrt{\exp \left(\Upsilon_{0}-\Upsilon_{t}\right)-1} \\
& \cong \sqrt{\exp \left\{\left[1+\ln \left(\frac{R_{0}}{R_{p l}}\right)\right]-\left[1+\ln \left(\frac{R_{t}}{R_{p l}}\right)\right]\right\}-1} \\
& \cong \sqrt{\exp \left\{\left(\ln \left(\frac{R_{0}}{R_{p l}}\right)-\ln \left(\frac{R_{t}}{R_{p l}}\right)\right)\right\}-1} \\
& \cong \sqrt{\exp \left\{\ln \left(\frac{R_{0}}{R_{t}}\right)\right\}-1} \cong \sqrt{\frac{R_{0}}{R_{t}}-1}
\end{aligned}
$$

to the squared cosmic temperature. The above relation (24) can be expressedas follows.

$$
Z \cong \sqrt{\frac{R_{0}}{R_{t}}-1} \cong \sqrt{\frac{T_{t}^{2}}{T_{0}^{2}}-1}
$$

Where $T_{t}$ is the past cosmic temperature and $T_{0}$ is the current cosmic temperature and $T_{t}>T_{0}$. For past higher cosmic temperatures, where $T_{t} \gg T_{0}$

$$
Z \cong \sqrt{\frac{T_{t}^{2}}{T_{0}^{2}}-1} \cong \frac{T_{t}}{T_{0}}
$$

This can be compared with the famous relation that is currently well believed by modern cosmologists.

$$
Z+1 \cong \frac{T_{t}}{T_{0}}
$$

Thus, it appears likely that at least a portion of the progressively higher redshift we observe with increasing look-back distance is a manifestation of gravitational time dilation. In addition, because of this inverse square relationship over very long distances, plots of proximal galactic redshifts per unit of distance observed would be expected to look relatively linear (as seen by the weaker telescopes of the 1920's and 1930's) and deep space galactic redshifts per unit of distance observed would be expected to clearly fall away from linearity, along with decreasing luminosity, as redshifts extend into the infrared range (as reported in 1998 Type Ia supernovae observations) (Perlmutter, S. et al. 1997). Such an effect may possibly create an illusion of dark energy whose current evidence is only marginal. The following graph (Figure 1), according to the above relation (24), shows expected observed cosmic redshift as a function of decreasing past cosmic radius $R_{t}$ pertaining to a particular astronomical observation. In this manner, increasingly greater redshifts would be expected to correspond with more distant galactic observations. The authors propose that something like this mathematical relationship could be useful in modeling the results of progressively deeper space observations. For data, see Table 2. In the last row of Table 2 the past cosmic radius $R_{t}$ and redshift of 1090 corresponding to the recombination temperature of $2990 \mathrm{~K}$ are correlated. Relations (24) and (28) closelyapproximate the recombination temperature of $3000 \mathrm{~K}$ and CMBR redshift 1100 believed to be related to formation of the first hydrogen atoms. Figure 1 may possibly provide an explanation for the nonlinearity of deep space Type Ia supernovae observations currently being attributed to "dark energy". Here it may be noted that, with reference to the suggestions proposed in the references of this paper, current universe seems to be expanding at constant rate and evidence for dark energy is only marginal. 


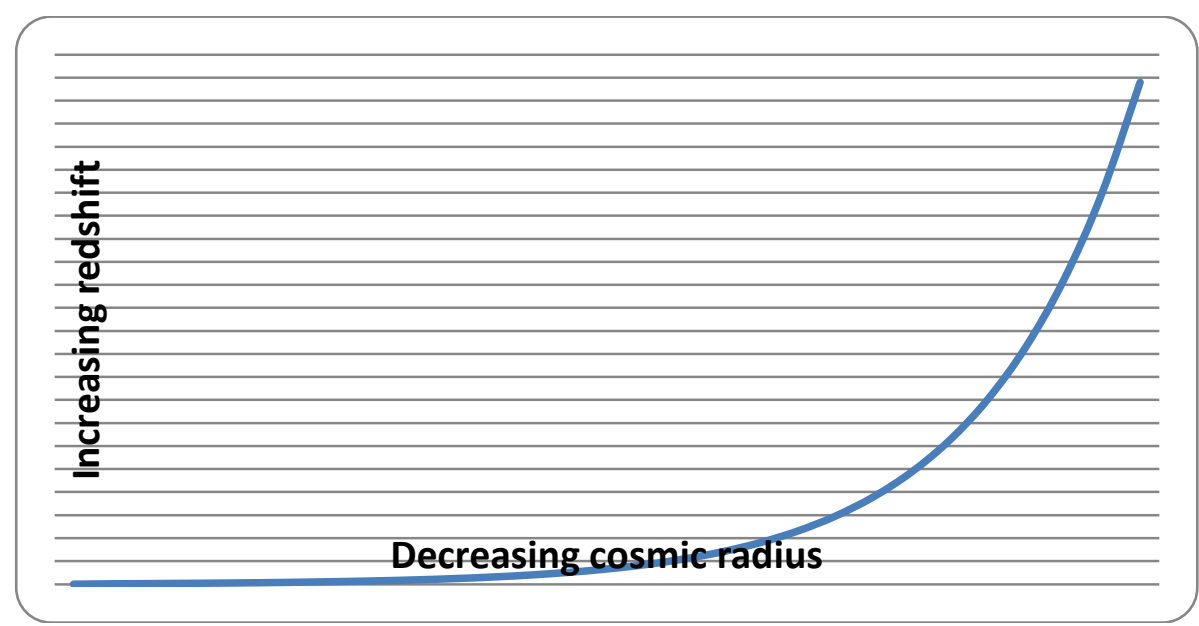

Fig. 1: Increasing Cosmic Redshift Vs Decreasing Past Cosmic Radius

Table 2:CosmicPhysical Parameters Obtained with above Relations

\begin{tabular}{|c|c|c|c|c|}
\hline $\begin{array}{l}\text { Assumed angular velocity } \\
(\mathrm{rad} / \mathrm{sec})\end{array}$ & $\begin{array}{c}\text { Estimated cosmic radius } \\
(\mathrm{m})\end{array}$ & Estimated galactic redshift & $\begin{array}{c}\text { Estimated cosmic tem- } \\
\text { perature }(\mathrm{K})\end{array}$ & $\begin{array}{c}\text { Estimated cosmic age } \\
\text { (Years) }\end{array}$ \\
\hline $1.49229 \mathrm{E}-20$ & $2.00894 \mathrm{E}+28$ & 0.0 & 2.75 & $3.10619 \mathrm{E}+14$ \\
\hline $2.45930 \mathrm{E}-20$ & $1.21902 \mathrm{E}+28$ & 0.8 & 3.52 & $1.87839 \mathrm{E}+14$ \\
\hline $4.05292 \mathrm{E}-20$ & $7.39695 \mathrm{E}+27$ & 1.3 & 4.52 & $1.13589 \mathrm{E}+14$ \\
\hline $6.67921 \mathrm{E}-20$ & 4.48844E+27 & 1.9 & 5.81 & $6.86886 \mathrm{E}+13$ \\
\hline $1.10073 \mathrm{E}-19$ & $2.72357 \mathrm{E}+27$ & 2.5 & 7.46 & $4.15361 \mathrm{E}+13$ \\
\hline $1.81401 \mathrm{E}-19$ & $1.65265 \mathrm{E}+27$ & 3.3 & 9.57 & $2.51167 \mathrm{E}+13$ \\
\hline $2.98949 \mathrm{E}-19$ & $1.00282 \mathrm{E}+27$ & 4.4 & 12.29 & $1.51878 \mathrm{E}+13$ \\
\hline $4.92668 \mathrm{E}-19$ & $6.08508 \mathrm{E}+26$ & 5.7 & 15.77 & $9.18374 \mathrm{E}+12$ \\
\hline 8.11917E-19 & $3.69240 \mathrm{E}+26$ & 7.3 & 20.25 & $5.55316 \mathrm{E}+12$ \\
\hline $1.33804 \mathrm{E}-18$ & $2.24054 \mathrm{E}+26$ & 9.4 & 25.99 & $3.35781 \mathrm{E}+12$ \\
\hline $2.20509 \mathrm{E}-18$ & $1.35955 \mathrm{E}+26$ & 12.1 & 33.37 & $2.03032 \mathrm{E}+12$ \\
\hline 3.63398E-18 & $8.24969 \mathrm{E}+25$ & 15.6 & 42.84 & $1.22764 \mathrm{E}+12$ \\
\hline $5.98881 \mathrm{E}-18$ & $5.00588 \mathrm{E}+25$ & 20.0 & 54.99 & $7.42282 \mathrm{E}+11$ \\
\hline $9.86955 \mathrm{E}-18$ & $3.03755 \mathrm{E}+25$ & 25.7 & 70.59 & $4.48810 \mathrm{E}+11$ \\
\hline $1.62650 \mathrm{E}-17$ & $1.84317 \mathrm{E}+25$ & 33.0 & 90.62 & $2.71363 \mathrm{E}+11$ \\
\hline $2.68048 \mathrm{E}-17$ & $1.11843 \mathrm{E}+25$ & 42.4 & 116.34 & $1.64071 \mathrm{E}+11$ \\
\hline $4.41742 \mathrm{E}-17$ & $6.78659 \mathrm{E}+24$ & 54.4 & 149.35 & $9.91995 \mathrm{E}+10$ \\
\hline 7.27991E-17 & $4.11808 \mathrm{E}+24$ & 69.8 & 191.73 & $5.99764 \mathrm{E}+10$ \\
\hline $1.19973 \mathrm{E}-16$ & $2.49883 \mathrm{E}+24$ & 89.7 & 246.13 & $3.62615 \mathrm{E}+10$ \\
\hline $1.97715 \mathrm{E}-16$ & $1.51628 \mathrm{E}+24$ & 115.1 & 315.97 & $2.19233 \mathrm{E}+10$ \\
\hline $3.25835 \mathrm{E}-16$ & $9.20074 \mathrm{E}+23$ & 147.8 & 405.62 & $1.32544 \mathrm{E}+10$ \\
\hline 5.36976E-16 & $5.58297 \mathrm{E}+23$ & 189.7 & 520.71 & $8.01323 \mathrm{E}+09$ \\
\hline $8.84937 \mathrm{E}-16$ & $3.38773 \mathrm{E}+23$ & 243.5 & 668.46 & $4.84451 \mathrm{E}+09$ \\
\hline $1.45838 \mathrm{E}-15$ & $2.05566 \mathrm{E}+23$ & 312.6 & 858.13 & $2.92877 \mathrm{E}+09$ \\
\hline $2.40340 \mathrm{E}-15$ & $1.24737 \mathrm{E}+23$ & 401.3 & 1101.62 & $1.77058 \mathrm{E}+09$ \\
\hline $3.96081 \mathrm{E}-15$ & $7.56897 \mathrm{E}+22$ & 515.2 & 1414.20 & $1.07039 \mathrm{E}+09$ \\
\hline $6.52741 \mathrm{E}-15$ & $4.59282 \mathrm{E}+22$ & 661.4 & 1815.47 & $6.47080 \mathrm{E}+08$ \\
\hline $1.07572 \mathrm{E}-14$ & $2.78691 \mathrm{E}+22$ & 849.0 & 2330.61 & $3.91174 \mathrm{E}+08$ \\
\hline $1.77278 \mathrm{E}-14$ & $1.69108 \mathrm{E}+22$ & 1089.9 & 2991.90 & $2.36470 \mathrm{E}+08$ \\
\hline
\end{tabular}

\section{Flatness and horizon problems and lambda term in this unified model}

Back ground history of Flatness problem: Ever since physicist Robert Dicke first made the observation (Dicke.R.H. 1970) in 1969 , cosmologists have been deeply puzzled as to how our universe appears to be expanding in a very precise way so as to perfectly balance out the attractive "force" of gravity. This is also what is meant by a "flat universe". In fact, as it was pointed out at the time, for such an apparent balance to be within observable error in the presumably opposing forces in the very early universe (within the first second after the Big Bang) must have been of equal magnitude to within one part in 1014. This has since been referred to as the "cosmological flatness problem." There is an excellent discussion of this problem in Alan Guth's book "The Inflationary Universe." As one of the pioneers and early proponents of the theory of cosmic inflation, Dr. Guth makes it very clear in his book that the flatness problem was a primary reason for which the theory of cosmic inflation was developed. a) Modern view of 'flatness' and its current status: Accordingto modern cosmology, criteria for 'flatness' is: sum of observable matter density, density of dark matter and density of dark energy should be equal to the critical density, $\left(\rho_{c r i}\right)_{0} \cong\left(3 H_{0}^{2} / 8 \pi G\right)$. Current cosmological observations clearly suggest that, evidence to cosmic acceleration is only marginal and at present universe is expanding at a constant rateand reference there in. If so currently believed 'dark energy that assumed to be constituting $\sim 68 \%$ of critical density' may be losing its identity in all respects. With reference to MOND, 'dark matter that assumed to be constituting $\sim 27 \%$ of critical density' seems to be losing its physical identity.Compared to 'dark energy', 'dark matter' seems to have some underlying particle physics back ground. But so far, no one could notice or find a 'characteristic particle' that can be called as the particle related with 'dark matter'. These points seriously cast doubt on the modern definition of 'flat universe' andseems to be reviewed at fundamental level.

b) Modern view of horizon problem: It had been a puzzle to cosmologists as to how a universe much larger than our own Hubble radius could have had any kind of causal connection to 
generate homogeneity. This has been called the "horizon problem." The theory of cosmic inflation, assumes an extremely brief period of superluminal hyper-rapid exponential expansion that believed to solve the flatness problem and the horizon problem simultaneously.

c) The authors opinion on flatness, homogeneity and horizon problems, primordial density fluctuations, the Lambda termand expansion speed

In this proposed model from the assumptions it is clear that, from the beginning of cosmic evolution, Universe starts expanding with light speed and decelerates. As time is passing, expansion speed follows

$v_{t} \cong\left\{1+\ln \left(\frac{\omega_{p l}}{\omega_{t}}\right)\right\}^{-1} c \cong\left\{1+\ln \left(\frac{R_{t}}{R_{p l}}\right)\right\}^{-1} c$.

Important points to be noted are:

1) Expansion speed seems to be very slowly decreasing by a factor $\left\{1+\ln \left(\frac{R_{t}}{R_{p l}}\right)\right\}$.

2) Expansion speed seems to be inversely proportional to cosmic size or cosmic age.

From table-1, in the beginning when the cosmic temperature was $2.74502 \mathrm{E}+31 \mathrm{~K}$, corresponding estimated expansion speed as $8.51656 \mathrm{E}+07 \mathrm{~m} / \mathrm{sec}$. Similarly, when the cosmic temperature was $8.68052 \mathrm{E}+30 \mathrm{~K}$, corresponding estimated expansion speed is $5.14869 \mathrm{E}+07 \mathrm{~m} / \mathrm{sec}$. Even though temperature is dropping by factor of 3.16, expansion speed ratio seems to be 1.65 . In the recent past, when the cosmic temperature was $8.68 \mathrm{~K}$, corresponding estimated expansion speed was $2.08221 \mathrm{E}+06 \mathrm{~m} / \mathrm{sec}$. When the cosmic temperature is $2.745 \mathrm{~K}$, corresponding estimated expansion speed is $2.04944 \mathrm{E}+06 \mathrm{~m} / \mathrm{sec}$. Even though temperature is dropping by a factor of 3.16 , expansion speed ratio seems to be 1.016only. If the current universe is very large, then such a small difference in expansion speed cannot be observed with current technology and more over it seems to simulate the effect of 'constant rate of expansion'. Thus this proposal apparently seems to be supported by the (very) recent cosmological observations that suggest 'constant rate of expansion' against "cosmic acceleration". In this context, the authors' proposed five assumptions can be given considerable importance. As the universe is shown to be decelerating at a very small rate, there is no need to consider the case of 'Flatness'. Whatever 'flatness' we believe it is an apparent effect of current cosmic (very slow) deceleration.

a) In this proposed model, in the first second of cosmic expansion, the universe expands from $1.6162 \times 10^{-35} \mathrm{~m}$ to $3.15 \times 10^{6} \mathrm{~m}$ and the ratio of expansion is $1.95 \times 10^{41}$. Similarly, in one second from the Planck scale, temperature drops from $9.67792 \times 10^{31} \mathrm{~K}$ to $2.19 \times 10^{11} \mathrm{~K}$ and the ratio of temperature drop in the first second is $4.423 \times 10^{20}$. Thus by considering these ratios the intended purpose of 'cosmic inflation effect' can be understood at fundamental level without requiring new physics. Not only that the estimated current age is 21400 times higher than the currently believed cosmic age. Thus past and current assumed effects of cosmic inflation can be understood. Clearly speaking, as cosmic time is very large, there is a lot scope for generation of ordered structures and smoothness in CMBR temperature.

b) In this proposed model, just crossing the Planck scale, at every stage of cosmic expansion,universe is confined to a size limited by $\left[R_{t} \cong c / \omega_{t} \cong G M_{t} / c^{2}\right]>\left[c / H_{0}\right]$. Clearly speaking, in this model current cosmic radius is 146 times more than the current Hubble radius. Thus the solution to the "horizon problem" is built into this model, not because the authors designed it with that intention, but because a universe bounded by $R_{0} \cong c / \omega_{0} \cong G M_{0} / c^{2}$ will always be causally connected. c) Cosmologists also postulate that primordial density perturbations resulting from primordial quantum fluctuations are responsible for the structure of the universe we see today. This also seems reasonable in this proposed model. Why because this proposed model is inherently connected with Planck scale. Planck scale itself may be responsible for the assumed primordial density fluctuations. Anyhow, in this context more study and additional mathematical modeling seems to be required.

d) If it is assumed that, $R_{t} \cong \frac{G M_{t}}{c^{2}} \cong \frac{c}{\omega_{t}}$, there is no need to think about Lambda term. Clearly speaking, at any stage of cosmic expansion, black hole universe having a size $\left(G M_{t} / c^{2}\right)$ will not collapse and will not fall down to its size. Th only fundamental question to be answered is: Why universe is evolving like a balck hole? Any how, qualitatively, Lambda term can be expressed with the following relation. At any stage of cosmic expansion,

$$
\left.\begin{array}{l}
\Lambda_{t}\left(\frac{c^{4}}{G}\right) \cong\left(\frac{9 c^{2} \omega_{t}^{2}}{20 \pi G}\right) \cong a T_{t}^{4} \text { and } \\
\Lambda_{t} \cong\left(\frac{9 c^{2} \omega_{t}^{2}}{20 \pi G}\right)\left(\frac{c^{4}}{G}\right)^{-1} \cong\left(\frac{9 \omega_{t}^{2}}{20 \pi c^{2}}\right) \\
\cong a T_{t}^{4}\left(\frac{c^{4}}{G}\right)^{-1} \cong \frac{G a T_{t}^{4}}{c^{4}}
\end{array}\right\}
$$

Here $\left(c^{4} / G\right)$ can be considered as the characteristic constant centripetal force of the light speed light speed rotating universe. Current magnitude of $\Lambda$ can be expressed as:

$$
\left.\begin{array}{rl}
\Lambda_{0} & \cong\left(\frac{9 c^{2} \omega_{0}^{2}}{20 \pi G}\right)\left(\frac{c^{4}}{G}\right)^{-1} \cong\left(\frac{9 \omega_{0}^{2}}{20 \pi c^{2}}\right) \\
& \cong \frac{G a T_{0}^{4}}{c^{4}} \cong 3.5415 \times 10^{-58} \mathrm{~m}^{-2}
\end{array}\right\}
$$

e) From the above assumptions and data it is very clear that, even though cosmic expansion speed is inversely proportional to cosmic age, at present universe is very slowly decelerating and thus resembles the effect of constant rate of expansion.

\section{Conclusion}

The authors stress the fact that, subject of cosmology is subjected to time to time cosmological observations, critical reviewson old concepts and new models of cosmology (U. V. S. Seshavatharam and Lakshminarayana S 2015, Tatum, E. T et al2015, Tatum, E.T 2015 a, 2015 b). With reference to current available data, qualitatively and quantitatively this proposed unified model can be analyzed theoretically in many possible ways. It may be true that, ratio of angular velocity and Hubble parameter is model dependent. Theoretically, compared to cosmic size and cosmic mass estimations, estimation of cosmic angular velocity seems to be easy and may yield workable models of cosmology. Now it seems essential to think and focus on developing 'observational methods' of cosmic angular velocity.

By considering the Planck scale, in this paper, the authors assumed that,

$H_{t} \cong \omega_{t}\left\{1+\ln \left(\omega_{p l} / \omega_{t}\right)\right\}$

And is for further critical study. As the assumed angular velocity is interlinked with Planck scale, its significance cannot be ignored. In future, either from 'academic interest' point of view or from 'serious research' point of view, 
1) By considering 'initial light speed expansion' and 'continuous light speed rotation' subject of cosmology can be simplified.

2) By guessing the 'Black hole radius' concept, subject of cosmology can be strengthened.

3) By guessing different ratios of angular velocity and Hubble parameter - different models of cosmology can be developed and a unified model of flat space (spherical) cosmology can be developed with respect to observational confirmation of the magnitude of angular velocity.

4) Quantum gravity point of view or Quantum cosmology point of view, relation (9) can be recommended for in depth study and analysis.

5) With reference to Hindu cosmology, modern cosmology can be reviewed at fundamental level.

\section{Acknowledgements}

Authors are very much thankful to Dr. Abhas Mithra and Dr. E. Terry Tatum. One of the authors, Seshavatharam U.V.S, is indebted to professors K.V. Krishna Murthy, Chairman, Institute of Scientific Research in Vedas (I-SERVE), Hyderabad, India and Shri K.V.R.S. Murthy, former scientist IICT (CSIR), Govt. of India, Director, Research and Development, I-SERVE, for their valuable guidance and great support in developing this subject.

\section{References}

[1] U.V.S. Seshavatharam and S. Lakshminarayana. Toy model of Evolving Spherical Cosmology with Flatness, Angular Velocity, Temperature and Redshift. Frontiers of Astronomy, Astrophysics and Cosmology, vol. 1, no. 2 pp.90-97 (2015)

[2] Ebenezer Burgess. Translation of the Surya Siddhanta, a text-book of Hindu Astronomy. Journal of the American Oriental Society 6, pp 141- 498 (1860) http://dx.doi.org/10.2307/592174.

[3] KedarNathShukla. Surya Siddhanta. D.K.Print world (P) Ltd. 1st edition. (2014)

[4] Nielsen. J.T et al. Marginal evidence for cosmic acceleration from Type IA supernovae. arXiv:1506.01354v2 (2015)

[5] Jun-Jie Wei et al. A Comparative Analysis of the Supernova Legacy Survey Sample with $\Lambda \mathrm{CDM}$ and the $\mathrm{Rh}=\mathrm{ct}$ Universe. The Astronomical Journal 149 102. (2015) http://dx.doi.org/10.1088/0004$6256 / 149 / 3 / 102$.

[6] F. Melia and R. S. Maier, Cosmic Chronometers in the $\mathrm{Rh}=\mathrm{ct}$ Universe. Mon.Not.Roy.Astron.Soc. 432, 2669 (2013)http://dx.doi.org/10.1093/mnras/stt596.

[7] Mitra, A. Energy of Einstein's static universe and its implications for the $\Lambda$ CDM cosmology. JCAP. 03: 007. (2013).

[8] Mitra, S. Bhattacharyya and N. Bhatt, LCDM Cosmology through the Lens of Einstein's Static Universe, the Mother of $\Lambda$. Int. J.Mod. phys. D 22, No.2, 1350012 (2013)http://dx.doi.org/10.1142/S0218271813500120.

[9] Steinhardt, P.J. The inflation debate: Is the theory at heart of modern cosmology deeply flawed? Scientific American, 304(4) pp. 18-25 (2011).http://dx.doi.org/10.1038/scientificamerican0411-36.

[10]Hawking, Stephen W. Quantum cosmology. In Hawking, Stephen W.; Israel, Werner. 300 Years of Gravitation. Cambridge University Press. pp. 631-651. (1987)

[11]W.M. Stuckey. The observable universe inside a black hole. Am. J. Phys. 62, 788 (1994)http://dx.doi.org/10.1119/1.17460.

[12]Tatum, E. T. U. V. S. Seshavatharam and S. Lakshminarayana. The basics of flat space cosmology. International journal of Astronomy and Astrophysics, 5, pp.116-124 (2015).

[13]Peebles, P. J. E. and Ratra, Bharat. The cosmological constant and dark energy. Reviews of Modern Physics 75 (2): 559-606 (2003)http://dx.doi.org/10.1103/RevModPhys.75.559.

[14]Guth, A.H. The Inflationary Universe. Basic Books, New York, US (1997).

[15]Guth, A.H.Inflationary universe: A possible solution to the horizon and flatness problems. Phys. Rev. D23: 347. (1981) http://dx.doi.org/10.1103/PhysRevD.23.347.

[16]Sivaram, C., and Arun, K. Primordial rotation of the universe, hydrodynamics, vortices and angular momenta of celestial objects. The Open Astronomy Journal, vol. 5, 7-11. (2012). http://dx.doi.org/10.2174/1874381101205010007.
[17] Yan Xu et al. Rings and Radial Waves in the Disk of the Milky Way. ApJ 801105 (2015) http://dx.doi.org/10.1088/0004-637X/801/2/105.

[18] Michael Longo. Detection of a dipole in the handedness of spiral galaxies with redshifts $\mathrm{Z} \sim 0.04$. Physics Letters B 699 (2011) 224 229 http://dx.doi.org/10.1016/j.physletb.2011.04.008.

[19]E.T. Whittaker, Spin in the universe, Yearbook of Roy. Soc. Edinburgh 5-13. (1945).

[20] Yuri N. Obukhov, on physical foundations and observational effects of cosmic Rotation. In Colloquium on Cosmic Rotation, Eds M. Scherfner, T. Chrobok and M. Shefaat (Wissenschaft und TechnikVerlag: Berlin, pp23-96, 2000. arXiv:astro-ph/0008106v1 (7 Aug 2000).

[21]Lukasz Andrzej Glinka. Global One-Dimensionality Conjecture within Quantum General Relativity. Gravitation and Cosmology 16(1), pp. 7-15 (2010). http://dx.doi.org/10.1134/S0202289310010020.

[22] Martin Bojowald. Quantum Cosmology. A Fundamental Description of the Universe. (Lecture Notes in Physics).' Vol. 835. Springer, (2011).

[23]Hawking, S.W. Particle creation by black holes. Commun. Math. Phys., v.43, 199-220. (1975).http://dx.doi.org/10.1007/BF02345020.

[24]Planck Collaboration: Planck 2015 results. XIII. Cosmological parameters. http://arxiv.org/abs/1502.01589. (2015).

[25]Fixsen, D.J. The Temperature of the Cosmic Micro-wave Background APJ. 707: 916. (2009).http://dx.doi.org/10.1088/0004-637X/707/2/916.

[26] Milgrom, M.A modification of the Newtonian dynamics as a possible alternative to the hidden mass hypothesis. Astrophys. J. 270: 365-370. (1983).http://dx.doi.org/10.1086/161130.

[27]J. R. Brownstein and J. W. Moffat. Galaxy rotation curves without non-baryonic dark matter. Astro-phys.J.636:721-741. (2006).http://dx.doi.org/10.1086/498208.

[28]Edmund A. Chadwick et al. A gravitational theoretical development supporting MOND. Phys.Rev. D88, 2, 024036. (2013).

[29]Hubble, E.P.A relation between distance and radial velocity among extra-galactic nebulae. PNAS. vol. 15: pp.168-173. (1929).http://dx.doi.org/10.1073/pnas.15.3.168.

[30]Hubble, E.P. The 200-inch telescope and some problems it may solve. PASP, 59: pp. 153-167. (1947).http://dx.doi.org/10.1086/125931.

[31]Perlmutter, S. et al. Measurements of the Cosmological Parameters $\Omega$ and $\Lambda$ from the First Seven Supernovae at $z \geq 0.35$. Astrophysical Journal 483 (2): 565. (1997).http://dx.doi.org/10.1086/304265.

[32]Dicke.R.H. Gravitation and the universe. American Philosophical Society.(1970).

[33]U. V. S. Seshavatharam and Lakshminarayana S. A brief report on scale independent quantum cosmology. Review of Advances in Physics Theories and Applications. 2(1): 1-9. (2015).

[34] Tatum, E. T. U. V. S. Seshavatharam and S. Lakshminarayana. Thermal radiation redshift in flat space cosmology. Journal of Applied Physical Science international. Vol 4, Issue 1, pp.18-26 (2015).

[35] Tatum, E.T. Could Our Universe Have Features of a Giant Black Hole? Journal of Cosmology, 25, 13063-13080. (2015a).

[36] Tatum, E.T. How a Black Hole Universe Theory Might Resolve Some Cosmological Conundrums. Journal of Cosmology, 25, 13081-13111. (2015b). 